\title{
Energy-Efficiency based Resource Allocation for the Scalar Broadcast Channel
}

\author{
Fabien Héliot, Muhammad Ali Imran, and Rahim Tafazolli \\ Centre for Communication Systems Research (CCSR), Faculty of Electronics \& Physical Sciences, \\ University of Surrey, Guildford GU2 7XH, UK. Email: F.Heliot@surrey.ac.uk
}

\begin{abstract}
Until recently, link adaptation and resource allocation for communication system relied extensively on the spectral efficiency as an optimization criterion. With the emergence of the energy efficiency (EE) as a key system design criterion, resource allocation based on EE is becoming of great importance. In this paper, we propose an optimal $\mathbf{E E}$ based resource allocation method for the scalar broadcast channel (BC-S). We introduce our EE framework, which includes an EE metric as well as a realistic power consumption model for the base station, and utilizes it for formulating our EE based optimization problem subject to a power as well as fairness constraints. We then prove the convexity of this problem and compare our EE based resource allocation method against two other methods, i.e. one based on sum-rate and one based on fairness. Results indicate that our method provides large $\mathrm{EE}$ improvement in comparison with the two other methods by significantly reducing the total consumed power. Moreover, they show that near-optimal EE and average fairness can be achieved simultaneously over the BS-C channel.
\end{abstract}

Index Terms-Energy efficiency, resource allocation, scalar broadcast channel, realistic power model.

\section{INTRODUCTION}

Energy efficiency (EE) is gradually becoming a key design criterion, along with the spectral efficiency (SE), for developing the next generation of reliable and low-power consumption communication network, in a context of ever increasing energy demand and price. As a research field, EE is quite mature regarding power-limited applications such as battery-driven system [1], e.g. mobile terminal, under acoustic telemetry [2], or wireless ad-hoc and sensor networks [3], [4]. However, as far as communication network is concerned, it can be considered as a new frontier, bringing new issues that are beginning to be addressed [5], [6]. This shift of focus in the research agenda from power-limited to power-unlimited applications is mainly driven by two factors: environmental, i.e. reducing the carbon footprint of communication system; commercial, i.e. reducing network operator ever-growing operational cost.

Link adaptation and resource allocation are powerful tools for improving the performance of communication system. In the past, SE was the criterion of choice for designing communication system and as a result sum-rate based optimization subject to a total power constraint has been extensively utilized for efficiently allocating resources. In order to make the resource allocation process fairer and allow for quality of service (QoS), fairness has also been used as a criterion but still in conjunction with SE. With the recent rise of the EE as a key performance evaluation criterion, resource allocation based on EE is becoming very popular, especially in the uplink of a single-cell system for increasing the battery autonomy of the user terminal (UT) [7]-[9]. The work in [8] propose a link adaptation method based on EE for the uplink of an orthogonal multi-carrier system operating over the additive white Gaussian noise (AWGN) channel for saving UT energy. In [9], this method has been refined for the same scenario but with more realistic assumptions on the circuit power and amplifier efficiency at the UT. As we previously mentioned, we are currently witnessing a shift of research focus from battery-limited to unlimited power applications and resource allocation is not immune from this trend. For instance, the work in [10] recently introduced a framework for optimizing the EE in the downlink of a single-cell system. This work focus on how to optimize the EE at the base station (BS) through power control in the case of elastic traffic. These works assume orthogonal subcarrier and thus do not account for interference, which greatly simplifies their problem formulations. Moreover, the works of [8] and [9] are user-centric and fail to address the EE of the single-cell system as a whole.

In this paper, we propose an optimal resource allocation method, i.e. power and rate allocations, for the scalar broadcast channel (BC-S) in terms of the EE. In other words, we address the problem of optimizing the EE in the downlink of a singlecell system as in [10], but in presence of interference and by considering the total energy consumed within the cell. In Section II, we first recall the BC-S model, the per-user SE formulation over this channel and show how to define the total transmit power at the BS as a function of the SE. We also introduce our EE framework that includes an EE metric, i.e. the Joule per bit, and a realistic power consumption model (PCM) for the BS [11]. In Section III, we then formulate our objective function for optimizing the $\mathrm{EE}$ as the function of the SE and prove its convexity by using the secondorder condition [12]. Next, we define our main problem, i.e. minimizing the energy consumption subject to a total power constraint, and then modify it to incorporate fairness through the Jain's fairness index [13]. In Section IV, we compare our EE based resource allocation method against the traditional methods based on sum-rate and fairness. Results show that our method provides large EE improvement via a significant reduction of the total consumed power in comparison with the two other methods. Moreover, they show that near-optimal $\mathrm{EE}$ and average fairness can be achieved at the same time. Conclusions are finally drawn in Section V. 


\section{SYSTEM AND POWER CONSUMPTION MODELS}

\section{A. System model}

We consider the downlink of a single-cell single-carrier single-antenna multi-user system, or equivalently the BC$\mathrm{S}$ model. A BS broadcasts a signal $x=\sum_{k=1}^{K} \sqrt{p_{k}} s_{k}$ to each of the $K$ users in its cell, with $s_{k}$ being the $k$-th user unit normalized transmit symbol and $p_{k}$ being the $k$-th user transmit power such that

$$
\mathrm{E}\left\{x x^{\dagger}\right\}=\sum_{k=1}^{K} p_{k}=P
$$

where $P$ is the total transmit power at the $\mathrm{BS}, \mathrm{E}\{$.$\} stands$ for the expectation and $\{.\}^{\dagger}$ is the conjugate operator. At each user receiver, the signal $x$ is received as $y_{k}=h_{k} x+n_{k}$, where $h_{k}$ represents the $k$-th user channel and $n_{k}$ is a zero-mean complex Gaussian variable with variance $N_{k}$ that accounts for the $k$-th user AWGN. Without loss of generality, we assume that $N_{k}=N=N_{0} W$ for all $k \in\{1, \ldots, K\}$, where $N_{0}$ is the noise power spectral density and $W$ is the bandwidth of the system. Assuming that dirty paper coding [14] is employed at the BS and that the users are ordered as in [15], i.e. the user with the strongest channel is denoted as user 1 and it does not see the interference from other users, the SE of the $k$-th user can be expressed as [15]

$$
S_{k}=\log _{2}\left(1+\frac{g_{k} p_{k}}{N \Gamma+g_{k} \sum_{j=1}^{k-1} p_{j}}\right) .
$$

Conversely from (2), we can express $p_{k}$ as

$$
p_{k}=\left(2^{S_{k}}-1\right)\left(\sum_{j=1}^{k-1} p_{j}+g_{k}^{-1} N \Gamma\right)
$$

where $g_{k}=\left|h_{k}\right|^{2}$ is the $k$-th user channel gain, $S_{k}=R_{k} / W$ with $R_{k}$ being the $k$-th user rate, and $\Gamma$ denotes the SNR gap between the channel capacity and the performance of a practical coding and modulation scheme as in [9]. We assume that $g_{1} \geq g_{2} \geq \ldots \geq g_{K}>0$, or conversely that $g_{K}^{-1} \geq$ $g_{K-1}^{-1} \geq \ldots \geq g_{1}^{-1}>0$, and define $\alpha_{k}=g_{K+1-k}^{-1}-g_{K-k}^{-1} \geq 0$ for $k \in\{1, \ldots, K-1\}$ and $\alpha_{K}=g_{1}^{-1}>0$. Inserting (3) into (1), the total transmit power can be reformulated as

$$
P=N \Gamma\left[-g_{K}^{-1}+\sum_{k=1}^{K} \alpha_{k} \prod_{j=1}^{k} e^{X_{j}}\right],
$$

where $X_{j}=\ln (2) S_{K+1-j}$ for $j \in\{1, \ldots, K\}$.

\section{B. Energy efficiency framework}

In order to minimize the energy consumption, or equivalently maximize the EE, of the multi-user system introduced in the previous subsection, we must first define the metric that will act as our objective function. In communication, the energy consumption is traditionally expressed in terms of energy-per-bit, which indicates how much energy is consumed by the system for transmitting bits. In a single-cell system, it can simply be defined as the ratio of the total consumed power within the cell, $P_{\mathrm{T}}$, to the sum of all the user rates in this cell, $\Sigma_{R}=\sum_{k=1}^{K} R_{k}$, such that $\Sigma_{E_{b}}=P_{\mathrm{T}} / \Sigma_{R}$, or equivalently

$$
\Sigma_{E_{b}}=\frac{P_{\mathrm{T}}}{W \sum_{k=1}^{K} S_{k}}
$$

when assuming that all users utilize the same bandwidth $W$.

The total consumed power by the system is obviously a key factor for determining how energy efficient is a system. In a conventional cellular system most of this power is consumed by the BS. A BS itself includes various components such as a transceiver, a power amplifier, a baseband interface, a signal processing unit, a power supply regulator, a cooling system, etc., and each of these components drains some power from the power socket. Recently in [11], a comprehensive power consumption analysis of the main power-hungry components of a BS has been carried out and, as a result, a realistic BS PCM has been proposed for various types of BS, which takes into account the non-linearity of the power amplifier. However, the relation between the relative radio frequency (RF) output power and BS power consumption is nearly linear [11] and, consequently, a linear abstraction of this model has been defined as [11]

$$
P_{\mathrm{BS}}=\Delta_{P} P+P_{0},
$$

where $\Delta_{P}$ and $P_{0}$ are the slope and overhead power of the PCM, respectively. In addition, $P \in\left[0, P_{\max }\right]$ with $P_{\max }$ being the maximum RF output power. Thus, the total consumed power for the downlink of a single-cell single-antenna multiuser system can be expressed as

$$
P_{\mathrm{T}}=\Delta_{P} P+P_{\mathrm{c}},
$$

where $P_{\mathrm{c}}=P_{0}+K P_{\mathrm{UT}}$ and $P_{\mathrm{UT}}$ is the consumed power by each UT for reception and processing.

\section{ENERGY CONSUMPTION MINIMIZATION}

Having defined $P_{\mathrm{T}}$ in (7) as a function of the transit power $P$ and having formulated $P$ in (4) as a function of the user SEs, we can re-expressed $\Sigma_{E_{b}}$ in (4) solely as a function of the user SEs, as follows

$$
\Sigma_{E_{b}}(\mathbf{X})=A \frac{f(\mathbf{X})}{g(\mathbf{X})}=A\left(\frac{\alpha_{0}+\sum_{k=1}^{K} \alpha_{k} \prod_{j=1}^{k} e^{X_{j}}}{\sum_{k=1}^{K} X_{k}}\right),
$$

where $\mathbf{X}=\left[X_{1}, \ldots, X_{K}\right] \succeq 0, A=\ln (2) W^{-1} N \Gamma \Delta_{P}$ and $\alpha_{0}=\frac{P_{\mathrm{c}}}{N \Gamma \Delta_{P}}-g_{K}^{-1}$. The function $1 / g(\mathbf{X})=\left(\sum_{k=1}^{K} X_{k}\right)^{-1}$ is clearly convex for $X_{k} \geq 0$ and, moreover, the function $f(\mathbf{X})=\alpha_{0}+\sum_{k=1}^{K} \alpha_{k} \prod_{j=1}^{k} e^{X_{j}}$ is also surely convex as long as $\alpha_{0} \geq 0$. However, it is not straightforward to conclude on the convexity of $\Sigma_{E_{b}}$ since the product of two convex functions is not necessarily convex because a convex function is not necessarily a log-convex function [12]. As long as $g>0$ then $-\ln (g)$ is convex and, hence, $g$ is log-convex. In turn, it implies that at least one $X_{k}$ variable must be strictly greater than zero or equivalently that there is always an active user, and we set this user to be user 1 such that $X_{K}=\ln (2) S_{1}>0$. 
Showing that $f$ is also log-convex under certain condition is not as straightforward as for $g$ and we prove it by using the second-order condition in the following subsections; first for the case of $K=2$ and then for the general case.

\section{A. Case of $K=2$}

Proof: The Hessian matrix of the natural logarithm of $f$, i.e. $H(\ln (f))$, can be defined as follows

$$
H(\ln (f))=\left(\begin{array}{cc}
\frac{\partial^{2} \ln (f)}{\partial X_{1}^{2}} & \frac{\partial^{2} \ln (f)}{\partial X_{1} \partial X_{2}} \\
\frac{\partial^{2} \ln (f)}{\partial X_{2} \partial X_{1}} & \frac{\partial^{2} \ln (f)}{\partial X_{2}^{2}}
\end{array}\right) .
$$

After straightforward computations, (9) can be simplified as

$$
H(\ln (f))=\frac{1}{f^{2}}\left(\begin{array}{c}
\frac{\partial f}{\partial X_{1}}\left(f-\frac{\partial f}{\partial X_{1}}\right) \\
\frac{\partial f}{\partial X_{2}}\left(f-\frac{\partial f}{\partial X_{1}}\right) \\
\frac{\partial f}{\partial X_{2}}\left(f-\frac{\partial f}{\partial X_{1}}\right) \frac{\partial f}{\partial X_{2}}\left(f-\frac{\partial f}{\partial X_{2}}\right)
\end{array}\right),
$$

where $\frac{\partial f}{\partial X_{1}}=f-\alpha_{0}$ and $\frac{\partial f}{\partial X_{2}}=f-\alpha_{0}-\alpha_{1} e^{X_{1}}$. Let $\mathbf{y}=\left[y_{1}, y_{2}, \ldots, y_{K}\right], \mathbf{y} \in \mathbb{R}^{K}$, the second-order condition [12] states that if $H(\ln (f))$ exists and $\beta=\mathbf{y} H(\ln (f)) \mathbf{y}^{\dagger} \geq 0$, i.e., $H(\ln (f))$ is positive semi-definite, then $f$ would be $\log$ convex. Using (10), we can express and simplify $f^{2} \beta$ as follows

$$
\begin{aligned}
f^{2} \beta & =y_{1}^{2} \alpha_{0}\left(\alpha_{1} e^{X_{1}}+\alpha_{2} e^{X_{1}+X_{2}}\right)+y_{2}^{2}\left(\alpha_{0}+\alpha_{1} e^{X_{1}}\right) \\
& \times \alpha_{2} e^{X_{1}+X_{2}}+2 y_{1} y_{2} \alpha_{0} \alpha_{2} e^{X_{1}+X_{2}} \\
& =y_{1}^{2} \alpha_{0} \alpha_{1} e^{X_{1}}+y_{2}^{2} \alpha_{1} \alpha_{2} e^{2 X_{1}+X_{2}}+\left(y_{1}+y_{2}\right)^{2} \\
& \times \alpha_{0} \alpha_{2} e^{X_{1}+X_{2}}
\end{aligned}
$$

Since $f^{2}>0$, it implies that $\beta \geq 0$ if $\alpha_{0} \alpha_{1} \geq 0, \alpha_{0} \alpha_{2} \geq 0$ and $\alpha_{1} \alpha_{2} \geq 0$. By definition $\alpha_{1} \geq 0$ and $\alpha_{2}>0$, hence, $\beta \geq 0$ and $f$ would be log-convex if $\alpha_{0} \geq 0$.

\section{B. General case}

Proof: In the general case, it can simply be proved that the $i$-th row element of the $j$-th column of the Hessian matrix of $\ln (f)$, i.e. $[H(\ln (f))]_{i, j}$, is given by

$$
[H(\ln (f))]_{i, j}=f^{-2} \frac{\partial f}{\partial X_{n}}\left(f-\frac{\partial f}{\partial X_{m}}\right),
$$

where $n=\max \{i, j\}, m=\min \{i, j\}$, and

$$
\frac{\partial f}{\partial X_{n}}=f-\left(\alpha_{0}+\sum_{k=1}^{n-1} \alpha_{k} \prod_{j=1}^{k} e^{X_{j}}\right) \text {. }
$$

Consequently, it can easily be proved that the matrix product $\beta=\mathbf{y} H(\ln (f)) \mathbf{y}^{\dagger}$ is equivalent to

$$
\beta=\sum_{k=0}^{K-1} \sum_{j=1}^{K-k}\left(\sum_{i=0}^{k} y_{j+i}\right)^{2} \alpha_{j-1} \alpha_{j+k} \prod_{u=1}^{j-1} e^{X_{u}} \prod_{v=1}^{j+k} e^{X_{v}}
$$

in the general case. Consequently, $\beta$ would be nonnegative and $f$ would be log-convex if all the possible combinations of $\alpha_{i} \alpha_{j}$ are nonnegative, $\forall i \in\{0, \ldots, K-1\}, \forall j \in\{1, \ldots, K\}$ and $j>i$. A necessary and sufficient condition is simply $\alpha_{0} \geq 0$ for $f$ to be log-convex.

Since it is well-know that the sum of two log-convex functions is itself a log-convex function, we can conclude that $\Sigma_{E_{b}}$ in (8) is a log-convex function and, hence, a convex function, as long as $\alpha_{K}, X_{K}>0$ and $\alpha_{0} \geq 0$. Conversely, note that $1 / \Sigma_{E_{b}}$ is log-concave and, hence, quasiconcave. Then, our optimization problem, i.e. minimizing the energy consumption while keeping $P \in\left[0, P_{\max }\right]$, is a convex optimization problem that can be formulated as

$$
\begin{array}{ll}
\min _{\mathbf{X}} & \Sigma_{E_{b}}(\mathbf{X}) \\
\text { s.t. } & \alpha_{k}, X_{k} \geq 0, \forall k \in\{1, \ldots, K-1\}, \\
& \alpha_{K}, X_{K}>0, \\
& N \Gamma\left[-g_{K}^{-1}+\sum_{k=1}^{K} \alpha_{k} \prod_{j=1}^{k} e^{X_{j}}\right] \leq P_{\max }
\end{array}
$$

Consequently, this problem can be solved by using classic method such as the interior-point method [12], which is integrated in the "fmincon" Matlab function. We denote this resource allocation method as $\mathrm{RA}_{\Sigma E_{b}}$ in the following.

\section{NUMERICAL RESULTS AND DISCUSSIONS}

In order to show that our resource allocation method reduces the energy-per-bit consumption of the system and study the trade-off between energy, rate and fairness, we benchmark our method against the sum-rate and min max fairness based resource allocation methods subject to a total power constraint, which we denote as $\mathrm{RA}_{\Sigma_{R}}$ and $\mathrm{RA}_{\mathcal{J}}$, respectively. They can be defined as

$$
\begin{array}{ll}
\max _{\mathbf{p}} & \Sigma_{R}=W \sum_{k=1}^{K} S_{k}\left(p_{k}\right) \\
\text { s.t. } & p_{k} \geq 0, \forall k \in\{1, \ldots, K\}, \text { and } \sum_{k=1}^{K} p_{k} \leq P_{\max }
\end{array}
$$

as well as

$$
\begin{array}{ll}
\max _{\mathbf{p}} & \min _{\left\{S_{k}\right\}}\left\{S_{k}\left(p_{k}\right)\right\} \\
\text { s.t. } & p_{k} \geq 0, \forall k \in\{1, \ldots, K\}, \text { and } \sum_{k=1}^{K} p_{k} \leq P_{\max },
\end{array}
$$

and, moreover, can easily be numerically solved by using the "fmincon" and "fminmax" functions in Matlab, respectively. Moreover, we compare the performance of these methods in terms of five metrics, the transmit power $P$ of the BS, the cell total consumed power $P_{\mathrm{T}}$, the cell total sum-rate $\Sigma_{R}$, the cell total energy-per-bit $\Sigma_{E_{b}}$, and the Jain's fairness index $\mathcal{J}$ given by [13]

$$
\mathcal{J}\left(S_{1}, \ldots, S_{K}\right)=\frac{\left(\sum_{k=1}^{K} S_{k}\right)^{2}}{K \sum_{k=1}^{K} S_{k}^{2}} .
$$

In our simulation, we assume that the $k$-th user channel gain is expressed as

$$
g_{k}=10^{\left(G_{\mathrm{TxRx}}-P L\left(r_{k}\right)\right) / 10}
$$


TABLE I

SIMULATION PARAMETER VALUES

\begin{tabular}{|c|c|c|c|}
\hline & & Parameters & Values \\
\hline \multirow{4}{*}{$\begin{array}{l}\mathrm{P} \\
\mathrm{C} \\
\mathrm{M}\end{array}$} & \multirow{3}{*}{ BS } & $\Delta_{P}$ & 7.6 \\
\hline & & $P_{0}$ & $435 \mathrm{~W}$ \\
\hline & & $P_{\max }$ & $40 \mathrm{~W}$ \\
\hline & UT [8] & $P_{\mathrm{UT}}$ & $100 \mathrm{~mW}$ \\
\hline \multirow{7}{*}{\multicolumn{2}{|c|}{$\begin{array}{c}\text { System } \\
\text { model } \\
{[16]}\end{array}$}} & $W$ & $10 \mathrm{MHz}$ \\
\hline & & $N_{0}$ & $-165.2 \mathrm{dBm} / \mathrm{Hz}$ \\
\hline & & $G_{T x R x}$ & $14 \mathrm{dBi}$ \\
\hline & & $P L_{\mathrm{LOS}}(d)$ & $31.24+24.2 \log 10(d), d$ in $\mathrm{m}$ \\
\hline & & $P L_{\mathrm{NLOS}}(d)$ & $3.14+42.8 \log 10(d), d$ in $\mathrm{m}$ \\
\hline & & $P_{\mathrm{LOS}}(d \leq 250 \mathrm{~m})$ & $\min \{18 / d, 1\}\left(1-e^{-d / 63}\right)+e^{-d / 63}$ \\
\hline & & $P_{\mathrm{LOS}}(d>250 \mathrm{~m})$ & $e^{(-(d-10) / 200)}$ \\
\hline
\end{tabular}

where $G_{\mathrm{TxRx}}$ is the antenna gain of the BS-UT transmission and $P L\left(d_{k}\right)=P_{\mathrm{LOS}}\left(d_{k}\right) P L_{\mathrm{LOS}}\left(d_{k}\right)+\left(1-P_{\mathrm{LOS}}\right) P L_{\mathrm{NLOS}}\left(d_{k}\right)$ is the path-loss as a function of the distance $d_{k}$ between the BS and the $k$-th user. In addition, $P_{\mathrm{LOS}}$ is the line-of-sight (LOS) probability, and $P L_{\mathrm{LOS}}\left(d_{k}\right)$ and $P L_{\mathrm{NLOS}}\left(d_{k}\right)$ are the LOS and non-LOS (NLOS) path-loss functions. We also assume as in [9] a capacity approaching coding and modulation scheme such that $\Gamma \simeq 1$. As far as the PCM is concerned, values of $\Delta_{P}$ and $P_{0}$ can be found in [11] but only for the two transmit antennas' case. In the single transmit antenna case, we obtain by using this PCM that the power consumptions calculated at $1 \%$ of the maximum and at the maximum are 383 $\mathrm{W}$ and $677 \mathrm{~W}$, respectively. Moreover, the relation between the relative RF output power and BS power consumption is nearly linear. Considering as in [11] that $P_{\max }=40 \mathrm{~W}$, we obtain $P_{0}=435 \mathrm{~W}$ and $\Delta_{P}=7.6$. Concerning the UT reception and processing power, we assume here that $P_{\mathrm{UT}}$ is in the order of the circuit power defined in [8] for the UT, i.e. $100 \mathrm{~mW}$. These PCM values and the system model parameter values, which have been obtained from [16], have been summarized in Table I for the reader convenience. Finally, note that the inequality $\alpha_{0} \geq 0$ holds as long as $d<3.89 \mathrm{~km}$ when considering the parameters of Table I, which is the case in our simulations.

In Fig. 1, we compare the resource allocation outcomes of our energy-per-bit minimization in (15), $\mathrm{RA}_{\Sigma E_{b}}$, against sumrate maximization in (16), $\mathrm{RA}_{\Sigma_{R}}$, and max-min fairness in (17), $\mathrm{RA}_{\mathcal{J}}$, in terms of the various metrics previously defined and the cell radius $r$ for $K=10$ users uniformly distributed within the cell. We also consider two modified versions of (15) that include a fairness constraint such that $\mathcal{J} \geq 0.5$ and $\mathcal{J}=1$, which we denote $\mathrm{RA}_{\Sigma E_{b}, \mathcal{J} \geq 0.5}$ and $\mathrm{RA}_{\Sigma E_{b}, \mathcal{J}=1}$, respectively. The results first show that our proposed resource allocation method $\mathrm{RA}_{\Sigma E_{b}}$ provides the lowest energy-per-bit consumption, or equivalently the best EE, as it was expected, by reducing drastically the total transmit power $P$ by about $90 \%$ in comparison with $\mathrm{RA}_{\Sigma_{R}}$ and $\mathrm{RA}_{\mathcal{J}}$. However, this comes at a cost of a lower sum-rate, about $30 \mathrm{Mbits} / \mathrm{s}$ in average, and level of fairness than $\mathrm{RA}_{\Sigma_{R}}$ and $\mathrm{RA} \mathcal{J}$, respectively. The results

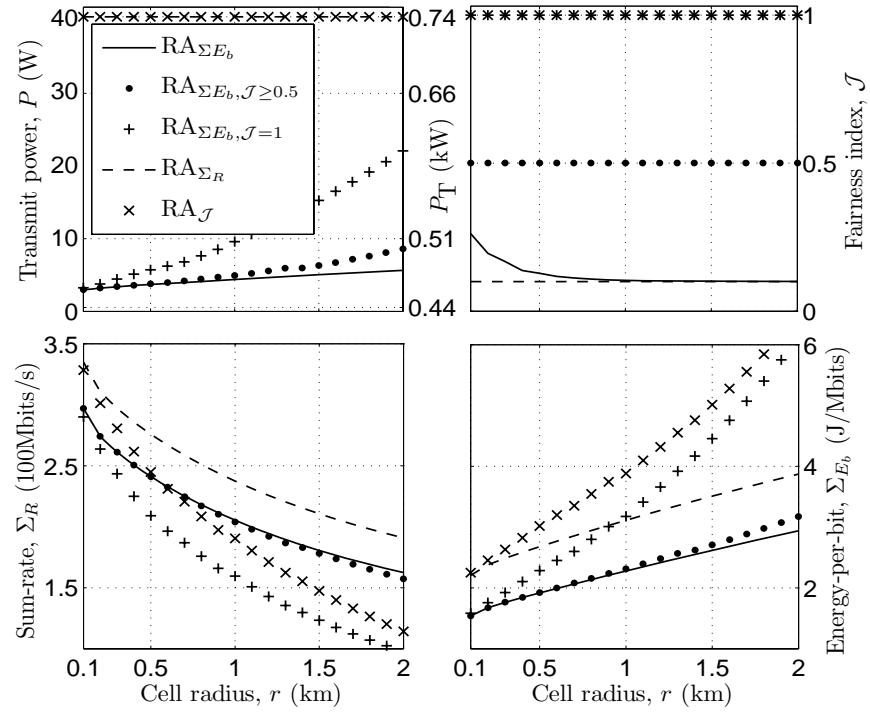

Fig. 1. Performance comparison of various resource allocation method for different metrics vs. cell rate radius $r$, when $K=10$ users.
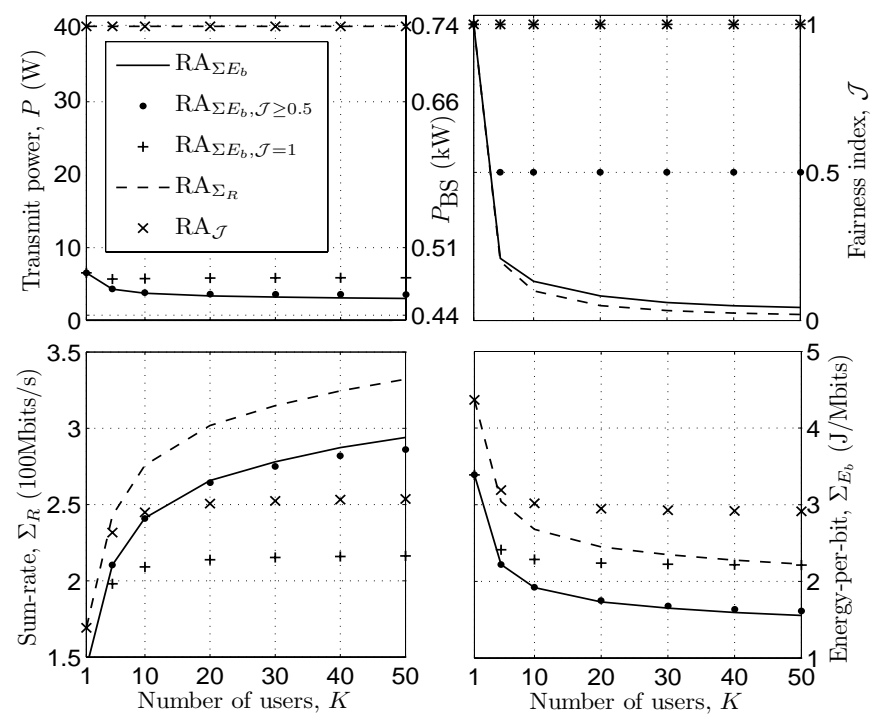

Fig. 2. Performance comparison of various resource allocation method for different metrics vs. number of users $K$, when $r=500 \mathrm{~m}$.

also indicate that a low energy-per-bit consumption is not incompatible with fairness, since low values for $P, P_{\mathrm{T}}$ and $\Sigma_{E_{b}}$ can be achieved by using $\mathrm{RA}_{\Sigma E_{b}, \mathcal{J}>0.5}$ while increasing the level of fairness from about 0.1 to 0.5 . Increasing further the level of fairness from 0.5 to 1 , low energy-per-bit consumption can still be achieved for small cell, i.e. $r \leq 500 \mathrm{~m}$.

In Fig. 2, we consider the same settings as in Fig. 1 and compare the same resource allocation methods but as a function of the number of users for a fixed cell radius of $r=500 \mathrm{~m}$. We still consider that the users are uniformly distributed and, consequently, increasing the number of users increases the sum-rate and decreases the energy consumption since as $K$ increases as the number of users close to the BS increases. Moreover, the resource allocation methods that 


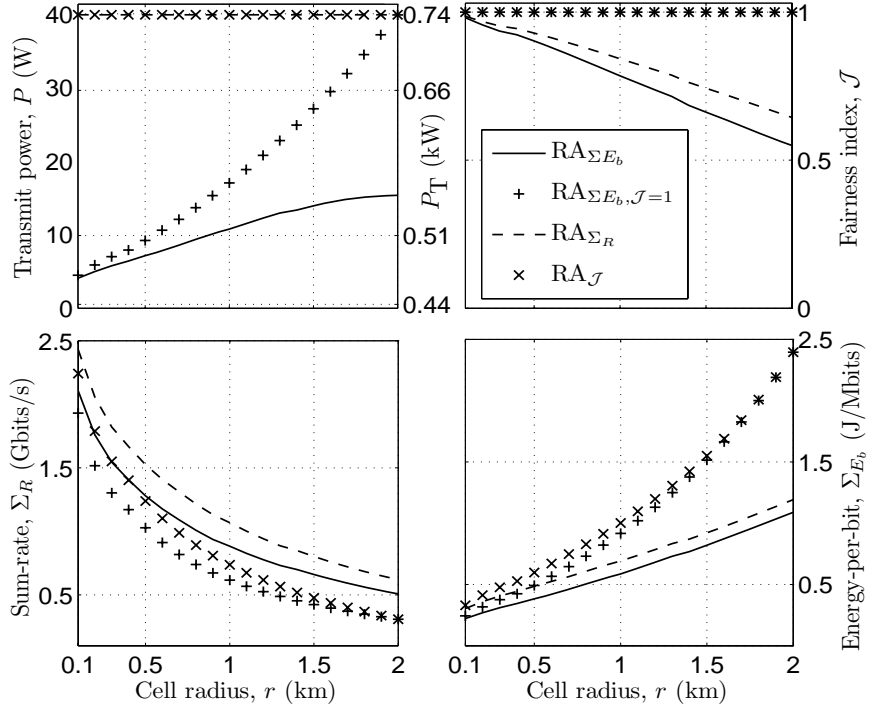

Fig. 3. Performance comparison of various resource allocation method when assuming no inter-user interference for different metrics vs. cell rate radius $r$, when $K=10$ users.

do not enforce fairness, i.e. $\mathrm{RA}_{\Sigma_{R}}$ and $\mathrm{RA}_{\Sigma E_{b}}$, see their level of fairness sharply reducing because these methods will favor the new users close to the BS to the detriment of the old users further to the BS for either increasing the sumrate or decreasing the energy consumption. As in Fig. 1, the results indicate that our $\mathrm{RA}_{\Sigma E_{b}}$ method outperforms both the $\mathrm{RA}_{\Sigma_{R}}$ and $\mathrm{RA}_{\mathcal{J}}$ methods in terms of energy-per-bit and power consumption. It can also be remarked that $\mathrm{RA}_{\Sigma E_{b}, \mathcal{J} \geq 0.5}$ allows us to increase the fairness while keeping a nearoptimal energy-per-bit consumption. Finally, we can achieve full fairness with a lower energy-per-bit than $\mathrm{RA}_{\Sigma_{R}}$ by using $\mathrm{RA}_{\Sigma E_{b}, \mathcal{J}=1}$ but at the cost of poor sum-rate performance.

In Fig. 3, we consider the same settings as in Fig. 1 and compare the same resource allocation methods when assuming no inter-user interference as in [8], [9] but for the downlink instead of the uplink scenario. Clearly, in absence of interuser interference the sum-rate is about 4 to 8 times larger, depending on the cell radius, than in the interference case. Consequently, the energy-per-bit consumption is also reduced by the same order of magnitude. The results confirm that $\mathrm{RA}_{\Sigma E_{b}}$ provides the lowest energy-per-bit consumption by reducing the total transmit and cell total consumed powers. However, in comparison with Fig. 1, the gap in terms of energy-per-bit consumption between $\mathrm{RA}_{\Sigma_{R}}$ and $\mathrm{RA}_{\Sigma E_{b}}$ is narrower and $\mathrm{RA}_{\Sigma_{R}}$ is fairer than $\mathrm{RA}_{\Sigma E_{b}}$ and, thus, it makes $\mathrm{RA}_{\Sigma E_{b}}$ more suitable for the interference case.

\section{CONCLUSION}

In this paper, an optimal EE based resource allocation method for the BC-S has been proposed when considering the total energy consumed within the cell. Based on our EE framework, we have formulated our objective function for opti- -mizing the EE as the function of the user SEs and prove its convexity. We have also defined our main problem, i.e. minimizing the energy consumption subject to a total power constraint, and then modify it to incorporate fairness. Next, we have compared our EE based resource allocation method against sum-rate and fairness based methods. Results indicated that our method always outperform the two other methods in terms of EE as well as transmit and cell total consumed powers. Moreover, they showed that near-optimal EE and average fairness can be achieved at the same time by using our method. In the future, we would like to extend our method to the vector broadcast channel.

\section{ACKNOWLEDGMENT}

The research leading to these results has received funding from the European Commission's Seventh Framework Programme FP7/2007-2013 under grant agreement n ${ }^{\circ}$ 247733project EARTH.

\section{REFERENCES}

[1] K. Lahiri, A. Raghunathan, S. Dey, and D. Panigrahi, "Battery-driven System Design: A New Frontier in Low Power Design," in Proc. Intl. Conf. on VLSI Design, Bangalore, India, Jan. 2002, pp. 261-267.

[2] H. M. Kwon and T. G. Birdsall, "Channel Capacity in Bits per Joule," IEEE J. Ocean. Eng., vol. OE-11, no. 1, pp. 97-99, Jan. 1986.

[3] C. Bae and W. E. Stark, "Energy and Bandwidth Efficiency in Wireless Networks," in Proc. IEEE ICCCAS, Guilin, China, Jun. 2006.

[4] S. Cui, A. J. Goldsmith, and A. Bahai, "Energy-Efficiency of MIMO and Cooperative MIMO Techniques in Sensor Networks," IEEE J. Sel. Areas Commun., vol. 22, no. 6, pp. 1089-1098, Aug. 2004.

[5] G. Auer et al., "Enablers for Energy Efficient Wireless Networks," in Proc. IEEE VTC-Fall, Ottawa, Canada, Sep. 2010, pp. 1-5.

[6] L. M. Correia et. al, "Challenges and Enabling Technologies for Energy Aware Mobile Radio Networks," IEEE Commun. Mag., vol. 11, pp. 6672, Nov. 2010.

[7] F. Meshkati, H. V. Poor, S. C. Schwartz, and N. B. Mandayam, "An Energy-Efficient Approach to Power Control and Receiver Design in Wireless Networks," IEEE Trans. Commun., vol. 5, no. 1, pp. 33063315, Nov. 2006

[8] G. Miao, N. Himayat, and G. Y. Li, "Energy-Efficient Link Adaptation in Frequency-Selective Channels," IEEE Trans. Commun., vol. 58, no. 2. pp. 545-554, Feb. 2010.

[9] C. Isheden and G. P. Fettweis, "Energy-Efficient Multi-Carrier Link Adaptation with Sum Rate-Dependent Circuit Power," in Proc. IEEE Globecom, Miami, USA, Dec. 2010, pp. 1-6.

[10] Z. Chong and E. Jorswieck, "Analytical Foundation for Energy Efficiency Optimisation in Cellular Networks with Elastic Traffic," in Proc. Mobilight 2011, Bilao, Spain, May 2011.

[11] G. Auer et al., "D2.3: Energy Efficiency Analysis of the Reference Systems, Areas of Improvements and Target Breakdown," INFSO-ICT247733 EARTH (Energy Aware Radio and NeTwork TecHnologies), Tech. Rep., Nov. 2010.

[12] S. Boyd and L. Vandenberghe, Convex Optimization. Cambridge, UK Cambridge Univ. Press, 2004.

[13] R. Jain, D. M. Chiuand, and W. Hawe, "A Quantitative Measure of Fairness and Discrimination for Resource Allocation in Shared Systems," DEC Research Report, Tech. Rep. TR-301, 1984.

[14] M. H. M. Costa, "Writing on Dirty Paper," IEEE Trans. Inf. Theory, vol. 27, no. 3, pp. 508-511, Jul. 1981.

[15] P. Viswanath and D. N. C. Tse, "Sum Capacity of the Vector Gaussian Broadcast Channel and Uplink-Downlink Duality," IEEE Trans. Inf. Theory, vol. 49, no. 8, pp. 1912-1921, Aug. 2003.

[16] A. Ambrosy and et al., "D2.2: Definition and Parameterization of Reference Systems and Scenarios," INFSO-ICT-247733 EARTH (Energy Aware Radio and NeTwork TecHnologies), Tech. Rep., Jun. 2010. 